\title{
IRREDUCIBLE REPRESENTATIONS OF THE POINCARE PARASUPERALGEBRA ${ }^{1}$ \\ A.G.Nikitin, V.V.Tretynyk \\ Institute of Mathematics, Ukrainian Academy of Sciences, Tereshenkivska str.3, Kiev-4, Ukraine
}

\section{ABSTRACT}

We describe explicitly all the irreducible unitary representations of the Poincaré parasuperalgebra,i.e., the parasupersymmetric extension of the Lie algebra of the Poincaré group. This parasuperalgebra includes as a particular case the usual Poincaré superalgebra and can serve as the group-theoretical foundation of parasupersymmetric quantum field theory.

\section{INTRODUCTION}

About twenty years ago there appears a new symmetry principle in physics which supposes existence of symmetry transformations mixing bosonic and fermionic states [1,2]. In addition to the usual Poincaré group generators the supplementary fermionic generators were taken into consideration, which connect fields with different statistics.

Supersymmetry provides a mechanism for the cancellation of the ultraviolet divergences in quantum field theory, makes it possible to unify space-time symmetries (i.e., Poincaré invariance) with internal symmetries [3] and opens additional ways to searching for unified field theories, including all the types of interactions [2].

Supersymmetric quantum field theory (SSQFT) [4] induced appearance of supersymmetric quantum mechanics (SSQM) [5]. Being very interesting by itself as a relative simple mathematical model of physical system with supersymmetry, SSQM stimulated more deep understanding of ordinary quantum mechanics and provided new ways for solving of some problems using, e.g., the concept of partner superpotential [6].

SSQM in its turn has been generalized [7] to parasupersymmetric quantum mechanics (PSSQM). The last deals with bosons and $p=2$ parafermions having parastatistical properties [8].

\footnotetext{
${ }^{1}$ The work was supported by the Ukrainian DKNT foundation for fundamental researches.
} 
The independent version of PSSQM corresponding to positive defined Hamiltonians was proposed in [9], the theories intermediate between SSQM and PSSQM were discussed in [10].

Rather recent theory called PSSQM awaked undoubted interest and stimulated appearance of a lot of papers, see [11] and papers cited therein. Parapotentials admitting Lie and non-Lie [12] symmetries were investigated in [13], hidden SU(3) symmetry of equations of PSSQM was established in [14].

The decisive step in developing of PSSQM was made by Beckers and Debergh [15] who asked for Poincaré invariance of the theory and formulated group-theoretical foundations of so-called parasupersymmetric quantum field theory (PSSQFT). This theory is a natural generalization of SSQFT, dealing with parastatistics instead of the usual Fermi or Bose statistics and with the Poincaré parasupergroup (or Poincaré parasuperalgebra, (PPSA)) instead of the Poincaré supergroup (or Poincaré superalgebra (PSA)). On the other hand, this theory is a relativistic extension of the PSSQM, preserving the main properties of the nonrelativistic parasupercharges.

Moreover, some dynamical models were proposed in [15], being parasupersymmetric analogues of the Wess-Zumino model [16].

We found it is necessary to analyze irreducible representations (IRs) of the PPSA. First, this is a way to make the group-theoretical fundamentals of the PSSFT, secondly, it enables us to generate a new view-point to the PSA which appears in our approach as a particular realization of the PPSA, thirdly, it indicates the specific role of the groups $\mathrm{SO}(3), \mathrm{SO}(5)$ and $\mathrm{SO}(2,3)$ in construction of internal super- and parasupersymmetries. Finally, the description of these IRs is an interesting mathematical problem admitting the exact and elegant solution.

Using the Wigner induced representations method we find all the IRs of the PPSA, for time-like, light-like and space- like four momenta. We also find covariant representations of the PPSA, which can have direct applications in PSSQFT.

\section{THE POINCARÉ PARASUPERALGE- BRA}

The Poincaré parasuperalgebra [15] includes ten generators $P_{\nu}, J_{\nu \sigma}$ of the Poincaré group, satisfying the usual commutation relations

$$
\left[P_{\mu}, P_{\nu}\right]=0, \quad\left[P_{\mu}, J_{\nu \sigma}\right]=i\left(g_{\mu \nu} P_{\sigma}-g_{\mu \sigma} P_{\nu}\right)
$$




$$
\begin{gathered}
{\left[J_{\mu \nu}, J_{\rho \sigma}\right]=i\left(g_{\mu \sigma} J_{\nu \rho}+g_{\nu \rho} J_{\mu \sigma}-g_{\mu \rho} J_{\nu \sigma}-g_{\nu \sigma} J_{\mu \rho}\right),} \\
J_{\mu \nu}=-J_{\nu \mu}, \quad \mu, \nu=0,1,2,3
\end{gathered}
$$

and four parasupercharges $Q_{A}, \bar{Q}_{A}(A=1,2)$ which satisfy the following double commutation relations

$$
\begin{gathered}
{\left[Q_{A},\left[Q_{B}, Q_{C}\right]\right]=\left[\bar{Q}_{A},\left[\bar{Q}_{B}, \bar{Q}_{C}\right]\right]=0} \\
{\left[Q_{A},\left[Q_{B}, \bar{Q}_{C}\right]\right]=-4 Q_{B}\left(\sigma_{\mu}\right)_{A C} P^{\mu}} \\
{\left[\bar{Q}_{A},\left[Q_{B}, \bar{Q}_{C}\right]\right]=4 \bar{Q}_{C}\left(\sigma_{\mu}\right)_{B A} P^{\mu}}
\end{gathered}
$$

Here $\sigma_{\nu}$ are the Pauli matrices, (.) $)_{A C}$ are the corresponding matrix elements.

Furthermore parasupercharges commute with generators of the Poincaré group as Weyl spinors

$$
\begin{aligned}
& {\left[J_{\mu \nu}, Q_{A}\right]=-\frac{1}{2 i}\left(\sigma_{\mu \nu}\right)_{A B} Q_{B},\left[P_{\mu}, Q_{A}\right]=0} \\
& {\left[J_{\mu \nu}, \bar{Q}_{A}\right]=-\frac{1}{2 i}\left(\sigma_{\mu \nu}\right)_{A B}^{*} \bar{Q}_{B},\left[P_{\mu}, \bar{Q}_{A}\right]=0}
\end{aligned}
$$

where $\sigma_{\nu \sigma}=-\sigma_{\sigma \nu}=\sigma_{\nu} \sigma_{\sigma}$.

The PPSA is a direct (and natural) generalization of the PSA [2]. Indeed, the PSA also includes 14 elements satisfying (2.1), (2.3), but instead of (2.2) supercharges $Q_{A}, \bar{Q}_{A}$ satisfy the following anticommutation relations

$$
\begin{aligned}
& {\left[Q_{A}, Q_{B}\right]_{+}=Q_{A} Q_{B}+Q_{B} Q_{A}=0, \quad\left[\bar{Q}_{A}, \bar{Q}_{B}\right]_{+}=0,} \\
& {\left[Q_{A}, \bar{Q}_{B}\right]_{+}=2\left(\sigma_{\mu}\right)_{A B} P^{\mu} .}
\end{aligned}
$$

We can make sure that (2.2) is a mere consequence of (2.4), the converse is not true. Thus, the PSA is a particular case of the more general algebraic structure called PPSA, like the usual Fermi statistics is a particular case of the parastatistics [8]. Moreover, in analogy with the PSA, $P_{\sigma}$ and $J_{\sigma \lambda}$ are called even, but $Q_{A}, \bar{Q}_{A}$ are called odd elements of the PPSA.

Some representations of the PPSA were described in [15]. Here we present the complete description of all the IRs of the parasuperalgebra (2.1)-(2.3).

\section{CASIMIR OPERATORS AND CLASSIFI- CATION OF THE IRS}

To find the main Casimir operators of the PPSA it is convenient to introduce the following four-vector [5]

$$
B_{\mu}=W_{\mu}+X_{\mu}
$$


where $W_{\nu}$ is the Lubanski-Pauli vector

$$
W_{\mu}=\frac{1}{2} \varepsilon_{\mu \nu \rho \sigma} J^{\nu \rho} P^{\sigma}
$$

and $X_{\nu}$ are the following bilinear combinations of parasupercharges

$$
\begin{array}{ll}
X_{0}=\frac{1}{8}\left\{\left[Q_{1}, \bar{Q}_{1}\right]+\left[Q_{2}, \bar{Q}_{2}\right]\right\}, & X_{1}=\frac{1}{8}\left\{\left[Q_{1}, \bar{Q}_{2}\right]+\left[Q_{2}, \bar{Q}_{1}\right]\right\} \\
X_{2}=\frac{i}{8}\left\{\left[Q_{2}, \bar{Q}_{1}\right]+\left[\bar{Q}_{2}, Q_{1}\right]\right\}, & X_{3}=\frac{1}{8}\left\{\left[\bar{Q}_{1}, Q_{1}\right]+\left[Q_{2}, \bar{Q}_{2}\right]\right\}
\end{array}
$$

Using (2.1)-(2.3) we find the following commutation relations

$$
\begin{aligned}
& {\left[B_{\mu}, P_{\nu}\right]=0, \quad\left[B_{\mu}, J_{\nu \sigma}\right]=i\left(g_{\mu \nu} B_{\sigma}-g_{\mu \sigma} B_{\nu}\right),} \\
& {\left[B_{\mu}, Q_{A}\right]=\frac{1}{2} P_{\mu} Q_{A}, \quad\left[B_{\mu}, \bar{Q}_{A}\right]=-\frac{1}{2} P_{\mu} \bar{Q}_{A},} \\
& {\left[B_{\mu}, B_{\nu}\right]=i \varepsilon_{\mu \nu \rho \sigma} P^{\rho} B^{\sigma}}
\end{aligned}
$$

from which it follows that the operators

$$
C_{1}=P_{\mu} P^{\mu}, \quad C_{2}=P_{\mu} P^{\mu} B_{\nu} B^{\nu}-\left(B_{\mu} P^{\mu}\right)^{2}
$$

are the Casimir operators of the PPSA. Indeed, $C_{1}$ coincides with the usual Casimir operator of the Poincaré algebra commuting with $Q_{A}, \bar{Q}_{A}$ in accordance with (2.3). The second Casimir $C_{2}$ is essentially new and includes the Poincaré invariant operator $W_{\nu} W^{\nu}$ as a constituent part. Thus, an IR of the PPSA is in general reducible in respect to the Lie algebra of the Poincaré group.

We will search for representations of the algebra (2.1)- (2.3) in the momentum representations, thus the action of the displacement operators $P_{\nu}$ will reduce to multiplication by $p_{\nu},-\infty<p_{\nu}<\infty$. In this case for any fixed $p_{\nu}$ relations $(2.2),(3.4 \mathrm{~b})$ define the algebra of operators $B_{\nu}, Q_{A}$ and $\bar{Q}_{A}$ which is going to be the main object of our investigations.

As in the case of the ordinary Poincaré algebra $[17,19]$ we distinguish the three main classes of IRs corresponding the following values of $C_{1}$ :

I. $P_{\mu} P^{\mu}=M^{2}>0$

II. $P_{\mu} P^{\mu}=0$

III. $P_{\mu} P^{\mu}=-\eta^{2}<0$

We will see that IRs of the PPSA distinguish qualitatively for different values of $C_{1}$ enumerated in (3.6). Moreover, these classes can be subdivided in accordance with different origins of eigenvalues of the second Casimir $C_{2}$ 
and of additional Casimir operators existing in particular classes I-III of IRs.

\section{IRs OF CLASS I}

If (3.6a) is valid then there exist the additional Casimir operator $C_{3}=$ $P_{0} /\left|P_{0}\right|$ whose eigenvalues are $\varepsilon= \pm 1$. We restrict ourselves to considering IRs corresponding to $\varepsilon=+1$ (for the case $\varepsilon=-1$ refer to Section 8 ). In this case we can define "a Wigner little parasuperalgebra " (LPSA) associated with the time-like four-vector $P=(M, 0,0,0)$. We set

$$
B_{k}=W_{k}+X_{k}=-M S_{k}+X_{k} \equiv M j_{k}, \quad k=1,2,3
$$

and obtain from $(3.4 \mathrm{~b})$

$$
\begin{gathered}
{\left[B_{0}, Q_{A}\right]=\frac{M}{2} Q_{A}, \quad\left[B_{0}, \bar{Q}_{A}\right]=-\frac{M}{2} \bar{Q}_{A}} \\
{\left[j_{k}, Q_{A}\right]=\left[j_{k}, \bar{Q}_{A}\right]=0} \\
{\left[j_{k}, j_{j}\right]=i \varepsilon_{k j l} j_{l}}
\end{gathered}
$$

On the other hand we obtain from (2.2)

$$
\left[Q_{A},\left[\bar{Q}_{A}, Q_{B}\right]\right]=4 M Q_{B}, \quad\left[\bar{Q}_{A},\left[Q_{A}, \bar{Q}_{B}\right]\right]=4 M \bar{Q}_{B}
$$

the other double commutators of $Q_{A}$ and $\bar{Q}_{A}$ are equal to zero.

It follows from (4.5) that the relation (4.2) turns to identity if (4.5) is satisfied.

In accordance with (4.3)-(4.5) the LPSA reduces to the direct sum of the Lie algebra whose basis elements are $j_{a}$ and the algebra of operators $Q_{A}, \bar{Q}_{A}$ characterized by double commutation relations (4.5). Thus, to describe IRs of this LPSA it is sufficient to find all the IRs of the subalgebras (4.4) and (4.5). Indeed, let $\tilde{j}_{a}$ and $I_{j}$ are basis elements of an IR of the algebra (4.4) and the unit operator in the space of this IR and $Q_{A}^{\prime}, \bar{Q}_{A}^{\prime}$ and $I_{Q}$ are basis elements of an IR of the algebra (4.5) and the unit operator in the space of this IR. Then, setting

$$
j_{a}=\tilde{j}_{a} \otimes I_{Q}, \quad Q_{A}=I_{j} \otimes Q_{A}^{\prime}, \quad \bar{Q}_{A}=I_{j} \otimes \bar{Q}_{A}^{\prime}
$$

(where $\otimes$ denotes the direct (Kronecker) product) we come to the IR of the algebra (4.3)-(4.5). Moreover, such a correspondence is a homomorphism. 
Relations (4.4) define the Lie algebra $\mathrm{AO}(3)$ of the rotation group $\mathrm{O}(3)$. IRs of this algebra are labelled by integers or half integers $\mathrm{j}$ so that

$$
\tilde{j}_{1}^{2}+\tilde{j}_{2}^{2}+\tilde{j}_{3}^{2}=j(j+1)
$$

The corresponding basis elements $\tilde{j}_{a}$ are the square matrices of dimension $(2 j+1) \times(2 j+1)$ which can be chosen in the following form [18]

$$
\begin{gathered}
\left(\tilde{j}_{3}\right)_{a b}=\delta_{a b}(j+1-a), \quad a, b=1,2, \ldots 2 j+1, \\
\left(\tilde{j}_{1} \pm i \tilde{j}_{2}\right)_{a b}=\delta_{a b \pm 1} \sqrt{j(j+1)-(j-a+1)(j-a+1 \pm 1)}
\end{gathered}
$$

To find IRs of the algebra (4.5) we choose the new basis

$$
\begin{array}{ll}
Q_{1}=\sqrt{2 M}\left(S_{51}+i S_{52}\right), & \bar{Q}_{1}=\sqrt{2 M}\left(S_{51}-i S_{52}\right), \\
Q_{2}=\sqrt{2 M}\left(S_{53}+i S_{54}\right), & \bar{Q}_{2}=\sqrt{2 M}\left(S_{53}-i S_{54}\right)
\end{array}
$$

and use the following notations for commutators

$$
\begin{aligned}
& {\left[Q_{1}, \bar{Q}_{1}\right]=4 M S_{12}, \quad\left[Q_{2}, \bar{Q}_{2}\right]=4 M S_{34},} \\
& {\left[Q_{1}, \bar{Q}_{2}\right]=2 M\left(i S_{24}-i S_{31}+S_{14}-S_{23}\right),} \\
& {\left[Q_{2}, \bar{Q}_{1}\right]=2 M\left(i S_{31}-S_{23}+S_{14}-i S_{24}\right),} \\
& {\left[Q_{1}, Q_{2}\right]=-2 M\left(i S_{31}+i S_{24}+S_{14}+S_{24}\right),} \\
& {\left[\bar{Q}_{1}, \bar{Q}_{2}\right]=2 M\left(-i S_{31}-i S_{24}+S_{14}+S_{23}\right) .}
\end{aligned}
$$

Formulae (4.9),(4.10) are invertible,so that

$$
\begin{gathered}
S_{51}=\frac{1}{2 \sqrt{2 M}}\left(Q_{1}+\bar{Q}_{1}\right), \quad S_{52}=-\frac{i}{2 \sqrt{2 M}}\left(Q_{1}-\bar{Q}_{1}\right), \\
S_{53}=\frac{1}{2 \sqrt{2 M}}\left(Q_{2}+\bar{Q}_{2}\right), \quad S_{54}=-\frac{i}{2 \sqrt{2 M}}\left(Q_{2}-\bar{Q}_{2}\right), \\
S_{12}=\frac{1}{4 M}\left[Q_{1}, \bar{Q}_{1}\right], \quad S_{34}=\frac{1}{4 M}\left[Q_{2}, \bar{Q}_{2}\right], \\
S_{14}=\frac{1}{8 M}\left(\left[Q_{1}, \bar{Q}_{2}\right]+\left[Q_{2}, \bar{Q}_{1}\right]+\left[\bar{Q}_{1}, \bar{Q}_{2}\right]-\left[Q_{1}, Q_{2}\right]\right), \\
S_{23}=\frac{1}{8 M}\left(\left[\bar{Q}_{1}, \bar{Q}_{2}\right]-\left[Q_{1}, Q_{2}\right]-\left[Q_{1}, \bar{Q}_{2}\right]-\left[Q_{2}, \bar{Q}_{1}\right]\right), \\
S_{13}=-\frac{i}{8 M}\left(\left[Q_{1}, Q_{2}\right]+\left[\bar{Q}_{1}, Q_{2}\right]+\left[Q_{1}, \bar{Q}_{2}\right]+\left[\bar{Q}_{1}, \bar{Q}_{2}\right]\right) \\
S_{24}=\frac{i}{8 M}\left(\left[Q_{1}, Q_{2}\right]-\left[Q_{1}, \bar{Q}_{2}\right]-\left[\bar{Q}_{1}, Q_{2}\right]+\left[\bar{Q}_{1}, \bar{Q}_{2}\right]\right) .
\end{gathered}
$$

Using (4.5), (4.11) we immediately find the following commutation relations for $S_{k l}=-S_{l k}, k, l=1,2, \ldots 5$

$$
\left[S_{k l}, S_{m n}\right]=i\left(\delta_{k m} S_{l n}+\delta_{l n} S_{k m}-\delta_{k n} S_{l m}-\delta_{l m} S_{k n}\right),
$$


which characterize the Lie algebra $\mathrm{AO}(5)$ of the rotation group in 5dimensional space.

IRs of the algebra $\mathrm{AO}(5)$ are labelled by pairs of numbers $\left(n_{1}, n_{2}\right)$ both integer or half integer, moreover $n_{1} \geq n_{2}$ [18]. The corresponding basis elements are square matrices of dimension $N\left(n_{1}, n_{2}\right)$, where

$$
N\left(n_{1}, n_{2}\right)=\frac{1}{6}\left(n_{1}-n_{2}+1\right)\left(n_{1}+n_{2}+2\right)\left(2 n_{1}+3\right)\left(2 n_{2}+1\right) .
$$

For the explicit form of these matrices see Appendix.

Thus we proved that for $P_{\nu} P^{\nu}>0$ the LPSA reduces to the direct sum of the algebras $\mathrm{AO}(3)$ and $\mathrm{AO}(5)$

$\mathrm{LPSA}=\mathrm{AO}(3) \oplus \mathrm{AO}(5)$

It follows from the above that IRs of the PPSA of class I with positive sign of energy are labelled by the sets of numbers $\left(M, j, n_{1}, n_{2}\right)$. To find the explicit form of the corresponding basis elements of the PPSA we start with the exact form of the Lubanski-Pauli vector $W_{\nu}^{\prime}$ in the frame of reference where $P=(M, 0,0,0)$, which, in accordance with (3.2),(4.1),(4.10), can be given by the following relations

$$
W_{0}^{\prime}=0, \quad W_{a}^{\prime}=-M\left(j_{a}+\frac{1}{4} \varepsilon_{a b c} S_{b c}+\frac{1}{2} S_{4 a}\right) \equiv-M S_{a} .
$$

Here

$$
j_{a}=\tilde{j}_{a} \otimes I_{N\left(n_{1}, n_{2}\right)}, \quad S_{k l}=I_{2 j+1} \otimes \hat{S}_{k l},
$$

$\tilde{j}_{a}$ and $\hat{S}_{k l}$ are basis elements of the IRs $D(j)$ and $D\left(n_{1}, n_{2}\right)$ of the algebras $\mathrm{AO}(3)$ and $\mathrm{AO}(5)$, correspondingly, $I_{N\left(n_{1}, n_{2}\right)}$ and $I_{2 j+1}$ are the unit matrices of dimensions $N\left(n_{1}, n_{2}\right) \times N\left(n_{1}, n_{2}\right)$ and $(2 j+1) \times(2 j+1)$.

The corresponding parasupercharges are present in (4.9). With the help of Lorentz transformation we find the explicit form of the Lubanski-Pauli vector and parasupercharges in arbitrary frame of reference

$$
\begin{gathered}
W_{0}=p_{a} S_{a}, \quad W_{a}=\varepsilon M S_{a}+\frac{p_{a} S_{b} p_{b}}{(E+M)}, \\
Q_{1}=\frac{1}{\sqrt{E+M}}\left[\left(S_{51}+i S_{52}\right)\left(E+M+\varepsilon p_{3}\right)+\varepsilon\left(S_{53}+i S_{54}\right)\left(p_{1}-i p_{2}\right)\right], \\
Q_{2}=\frac{1}{\sqrt{E+M}}\left[\varepsilon\left(S_{51}+i S_{52}\right)\left(p_{1}+i p_{2}\right)+\left(S_{53}+i S_{54}\right)\left(E+M-\varepsilon p_{3}\right)\right]
\end{gathered}
$$




$$
\bar{Q}_{A}=Q_{A}^{+}
$$

where

$$
E=\sqrt{M^{2}+p^{2}}, \quad p^{2}=p_{1}^{2}+p_{2}^{2}+p_{3}^{2} .
$$

The explicit form of generators of the Poincaré group, corresponding to the Lubanski-Pauli vector (4.17), is well- known (see, e.g.,[19]), and given by the formulae

$$
\begin{gathered}
P_{0}=\varepsilon E, \quad P_{a}=p_{a}, \\
J_{a b}=x_{a} p_{b}-x_{b} p_{a}+\varepsilon_{a b c} S_{c}, \\
J_{0 a}=x_{0} p_{a}-\frac{i \varepsilon}{2}\left[\frac{\partial}{\partial p_{a}}, E\right]_{+}-\varepsilon \frac{\varepsilon_{a b c} p_{b} S_{c}}{E+M} .
\end{gathered}
$$

Thus, we have enumerated all the non-equivalent IRs of the PPSA of class I and find the explicit form of the corresponding basis elements, see (4.15), (4.18), (4.19).

\section{IRs OF CLASS II}

In this case we again have the additional Casimir $C_{3}=P_{0} /\left|P_{0}\right|=\varepsilon= \pm 1$. As before we consider the case $\varepsilon=+1$, refer to Section 8 for the other case.

To obtain the corresponding LPSA we choose the light-like four-vector $P=(M, 0,0, M)$. The corresponding algebra (2.2) reduces to the form

$$
\begin{array}{ll}
{\left[Q_{2},\left[\bar{Q}_{2}, Q_{2}\right]\right]=8 M Q_{2},} & {\left[\bar{Q}_{2},\left[Q_{2}, \bar{Q}_{2}\right]\right]=8 M \bar{Q}_{2},} \\
{\left[Q_{2},\left[\bar{Q}_{2}, Q_{1}\right]\right]=8 M Q_{1},} & {\left[\bar{Q}_{2},\left[Q_{2}, \bar{Q}_{1}\right]\right]=8 M \bar{Q}_{1},}
\end{array}
$$

the remaining double commutators equal to zero.

Let us start with (5.1). Denoting

$$
j_{1}=\frac{1}{4 \sqrt{M}}\left(Q_{2}+\bar{Q}_{2}\right), \quad j_{2}=\frac{i}{4 \sqrt{M}}\left(Q_{2}-\bar{Q}_{2}\right), \quad j_{3}=\frac{1}{8 M}\left[Q_{2}, \bar{Q}_{2}\right]
$$

we find that $j_{a}$ have to satisfy the relations (4.4), characterizing the algebra $\mathrm{AO}(3)$. The relations (5.3) are invertible, thus the algebra (5.1) reduces to the algebra $\mathrm{AO}(3)$. Then the relations (5.2) (completed by the zero double commutators) have not nontrivial solutions for $Q_{1}$ and $\bar{Q}_{1}$. So we come to the following general form of parasupercharges

$$
Q_{2}=2 \sqrt{M}\left(j_{1}-i j_{2}\right), \quad \bar{Q}_{2}=2 \sqrt{M}\left(j_{1}+i j_{2}\right), \quad Q_{1}=\bar{Q}_{1} \equiv 0,
$$

where $j_{a}$ are basis elements of the algebra $\mathrm{AO}(3)$. 
In accordance with (3.4b), (5.4), (4.4) we obtain

$$
\begin{gathered}
{\left[B_{0}, Q_{1}\right]=\frac{M}{2} Q_{1},\left[B_{0}, \bar{Q}_{1}\right]=-\frac{M}{2} \bar{Q}_{1}, B_{3}=B_{0}} \\
{\left[B_{0}, B_{1}\right]=i M B_{2},\left[B_{0}, B_{2}\right]=-i M B_{1},\left[B_{0}, B_{1}\right]=0 .}
\end{gathered}
$$

Defining

$$
\begin{gathered}
B_{0}=W_{0}+X_{0}=W_{0}+M j_{3} \equiv M\left(T_{0}-\frac{1}{2}\left(j-j_{3}\right)\right), \\
W_{0}=M\left(T_{0}-\frac{1}{2}\left(j+j_{3}\right)\right), \quad B_{1}=W_{1} \equiv T_{1}, \quad B_{2}=W_{2} \equiv T_{2},
\end{gathered}
$$

we obtain from $(5.5)$

$$
\begin{gathered}
{\left[T_{0}, T_{1}\right]=i T_{2}, \quad\left[T_{0}, T_{2}\right]=-i T_{1}, \quad\left[T_{1}, T_{2}\right]=0,} \\
{\left[T_{0}, j_{a}\right]=\left[T_{1}, j_{a}\right]=\left[T_{2}, j_{a}\right]=0 .}
\end{gathered}
$$

We see that LPSA reduces to the direct sum of the algebras $\mathrm{AO}(3)$ and $\mathrm{AE}(2)$, characterized by relations (4.4) and (5.7) correspondingly. In other words

$\mathrm{LPSA}=\mathrm{AE}(2) \oplus \mathrm{AO}(3)$.

IRs of the algebra $\mathrm{AE}(2)$ are of two kinds corresponding to zero and nonzero eigenvalues of the Casimir $C=T_{1}^{2}+T_{2}^{2}$. If

$C=T_{1}^{2}+T_{2}^{2}=0$

then

$T_{1}=T_{2}=0, T_{0}=\lambda$,

where $\lambda$ is an arbitrary (fixed) integer or half integer. If

$C=T_{1}^{2}+T_{2}^{2}=r^{2}>0$

the corresponding IRs are realized by infinite-dimensional matrices. Let $\mid r, n>$ is the eigenvector of the commuting operators $C$ and $T_{0}$, then

$$
\begin{gathered}
C\left|r, n>=r^{2}\right| r, n>, \quad T_{0}|r, n>=n| r, n>, \\
\left(T_{1} \pm i T_{2}\right)|r, n>=r| r, n \pm 1>.
\end{gathered}
$$

Thus IRs of the algebra (4.4), (5.7), (5.8) are labelled by pairs of numbers $(j, r)$ (or $(j, \lambda)$ if $r=0$ ). Denoting by $\mid j, \nu ; r, n>$ the common eigenvector of the commuting matrices $j^{2}, j_{3}, C, T_{0}$ and using (4.8), (5.11), (5.12) we can 
represent basis elements of IRs of this algebra in the form

$$
\begin{gathered}
j_{3}|j, \nu ; r, n>=\nu| j, \nu ; r, n>, \quad \nu=j, j-1, \ldots-j \\
\left(j_{1} \pm i j_{2}\right)|j, \nu ; r, n>=\sqrt{j(j+1)-\nu(\nu \pm 1)}| j, \nu \pm 1 ; r, n>, \\
T_{0}|j, \nu ; r, n>=n| j, \nu ; r, n>, \\
\left(T_{1} \pm i T_{2}\right)|j, \nu ; r, n>=r| j, \nu ; r, n \pm 1>, \\
\begin{cases}n=0, \pm 1, \pm 2, \ldots \text { or } n= \pm \frac{1}{2}, \pm \frac{3}{2}, \pm \frac{5}{2}, \ldots, & r \neq 0 \\
n=\lambda, & r=0 .\end{cases}
\end{gathered}
$$

Thus, we have found the explicit form of IRs of the operators $W_{\nu}, Q_{A}, \bar{Q}_{A}$ in the reference frame $P=(M, 0,0, M)$. To find these operators (and the corresponding generators $P_{\nu}, J_{\nu \sigma}$ ) in an arbitrary frame of reference it is sufficient to make the corresponding rotation transformation. As a result we obtain

$$
\begin{gathered}
Q_{1}=\frac{\sqrt{2}\left(-p_{1}+i p_{2}\right)}{\sqrt{p+p_{3}}}\left(j_{1}-i j_{2}\right), \quad \bar{Q}_{1}=\frac{\sqrt{2}\left(-p_{1}-i p_{2}\right)}{\sqrt{p+p_{3}}}\left(j_{1}+i j_{2}\right), \\
Q_{2}=\sqrt{2\left(p+p_{3}\right)}\left(j_{1}-i j_{2}\right), \quad \bar{Q}_{2}=\sqrt{2\left(p+p_{3}\right)}\left(j_{1}+i j_{2}\right), \\
P_{0}=\varepsilon p, \quad P_{a}=p_{a} \\
J_{a} b=x_{a} p_{b}-x_{b} p_{a}+\varepsilon_{a b c} \hat{T}_{0} \frac{p_{c}+\delta_{c 3} p}{p+p_{3}} \\
J_{0 a}=x_{0} p_{a}-\frac{1}{2} \varepsilon\left[p, x_{a}\right]_{+}+\frac{\varepsilon_{a b c} T_{b} p_{c}}{p^{2}}-\frac{\varepsilon_{a b c} p_{b} n_{c}\left(\varepsilon \hat{T}_{0} p^{2}-T_{a} p_{a}\right)}{p^{2}\left(p+p_{3}\right)}
\end{gathered}
$$

where

$$
p=\sqrt{p_{1}^{2}+p_{2}^{2}+p_{3}^{2}}, \mathbf{n}=(0,0,1), T_{3}=0, \hat{T}_{0}=T_{0}-\frac{1}{2}\left(j_{3}+j\right) .
$$

For the important for physics case $C=r^{2}=0$ (representations with discrete spin) formulae (5.15) are simplified and reduced to the form

$$
\begin{gathered}
P_{0}=\varepsilon p, \quad P_{a}=p_{a}, \\
J_{a} b=x_{a} p_{b}-x_{b} p_{a}+\frac{1}{2} \varepsilon_{a b c}\left(2 \lambda-j-j_{3}\right) \frac{p_{c}+\delta_{c 3} p}{p+p_{3}}, \\
J_{0 a}=x_{0} p_{a}-\frac{1}{2} \varepsilon\left[p, x_{a}\right]_{+}-\frac{\varepsilon}{2} \varepsilon_{a b c}\left(2 \lambda-j-j_{3}\right) \frac{p_{b} n_{c}}{p+p_{3}},
\end{gathered}
$$

where $\lambda$ and $j$ are arbitrary integers or half integers.

So IRs of the PPSA, belonging to class II with $P_{0}>0$, are labelled by the sets of numbers $(r, j), r \neq 0$ or $(\lambda, j)$ for $r=0$. The explicit form of the corresponding basis elements is given in (5.14)-(5.16),(5.13). 


\section{IRs OF CLASS III}

To obtain the corresponding LPSA we choose the space-like four-vector $P=(0,0,0, \eta)$. The corresponding double commutation relations $(2.2)$ reduce to the form

$$
\begin{aligned}
& {\left[Q_{1},\left[\bar{Q}_{1}, Q_{B}\right]\right]=-4 \eta Q_{B},\left[\bar{Q}_{1},\left[Q_{1}, \bar{Q}_{B}\right]\right]=-4 \eta \bar{Q}_{B}} \\
& {\left[Q_{2},\left[\bar{Q}_{2}, Q_{B}\right]\right]=4 \eta Q_{B},\left[\bar{Q}_{2},\left[Q_{2}, \bar{Q}_{B}\right]\right]=4 \eta \bar{Q}_{B},}
\end{aligned}
$$

the remaining double commutators are equal to zero. Moreover, denoting

$$
\begin{aligned}
& B_{0}=-J_{12} \eta+X_{0} \equiv \eta \tilde{J}_{12}, \\
& B_{1}=-J_{02} \eta+X_{1} \equiv \eta \tilde{J}_{01}, \\
& B_{2}=J_{01} \eta+X_{2} \equiv \eta \tilde{J}_{02}
\end{aligned}
$$

and remembering that $B_{3}=X_{3}$, we find from (3.4b), that

$$
\begin{gathered}
{\left[\tilde{J}_{\alpha \beta}, Q_{A}\right]=\left[\tilde{J}_{\alpha \beta}, \bar{Q}_{A}\right]=0, \quad \alpha, \beta=0,1,2,} \\
{\left[\tilde{J}_{\alpha \beta}, \tilde{J}_{\rho \sigma}\right]=i\left(g_{\alpha \sigma} \tilde{J}_{\beta \rho}+g_{\beta \rho} \tilde{J}_{\alpha \sigma}-g_{\alpha \rho} \tilde{J}_{\beta \sigma}-g_{\beta \sigma} \tilde{J}_{\alpha \rho}\right),}
\end{gathered}
$$

where

$$
g_{00}=-g_{11}=-g_{22}=1, \quad g_{\alpha \beta}=0, \quad \alpha \neq \beta .
$$

In accordance with (6.1)-(6.4) the LPSA corresponding to space-like momenta reduces to the direct sum of the algebra $\mathrm{AO}(1,2)$ (defined by relations (6.4)) and the algebra, defined by the double commutation relations (6.1). The last reduces to the algebra $\mathrm{AO}(2,3)$, if we define $Q_{A}, \bar{Q}_{A}$ and the corresponding commutators using the relations (4.9), (4.10) (with the new meaning of $M$, compare (3.6a) and (3.6c)). Indeed, in this case we immediately find that $S_{k l}$ have to satisfy the algebra $\mathrm{AO}(2,3)$. The corresponding commutation relations can be obtained from (4.12) by the change $\delta_{k l} \rightarrow-g_{k l}$, where

$$
g_{11}=g_{22}=-g_{33}=-g_{44}=-g_{55}=1, \quad g_{k l}=0, \quad k \neq l .
$$

Thus we make sure that the LPSA for representations of class III reduces to the direct sum of the algebras $\mathrm{AO}(1,2)$ and $\mathrm{AO}(2,3)$

$\mathrm{LPSA}=\mathrm{AO}(1,2) \oplus \mathrm{AO}(2,3)$

IRs of the algebra (6.6) can be constructed in analogy with (4.16). For IRs of the algebras $\mathrm{AO}(1,2)$ and $\mathrm{AO}(2,3)$ see,e.g., [20]. 
Starting with (6.2), (4.9), (3.1) and making the Lorentz transformation and the rotation corresponding to transition to an arbitrary frame of references, we find the corresponding basis elements of the PPSA in the form

$$
\begin{gathered}
P_{\mu}=p_{\mu}, J_{a b}=x_{a} p_{b}-x_{b} p_{a}+\tilde{S}_{a b}, \\
J_{0 a}=x_{0} p_{a}-\frac{1}{2}\left[x_{a}, p_{0}\right]_{+}+\tilde{S}_{0 a}, \\
J_{a 3}=x_{a} p_{3}-x_{3} p_{a}-\frac{\tilde{S}_{a b} p_{b}-\tilde{S}_{a 0} p_{0}}{p_{3}+\eta}, \\
J_{03}=x_{0} p_{3}-\frac{1}{2}\left[x_{3}, p_{0}\right]_{+}-\frac{\tilde{S}_{0 a} p_{a}}{p_{3}+\eta}, \\
Q_{1}=\frac{1}{\sqrt{\left(\eta+p_{3}\right)}}\left[\left(\eta+p_{3}-p_{0}\right)\left(S_{51}+i S_{52}\right)-\left(p_{1}-i p_{2}\right)\left(S_{53}+i S_{54}\right)\right], \\
Q_{2}=\frac{1}{\sqrt{\left(\eta+p_{3}\right)}}\left[\left(p_{1}+i p_{2}\right)\left(S_{51}+i S_{52}\right)+\left(\eta+p_{3}+p_{0}\right)\left(S_{53}+i S_{54}\right)\right], \\
\bar{Q}_{1}=\frac{1}{\sqrt{\left(\eta+p_{3}\right)}}\left[\left(\eta+p_{3}-p_{0}\right)\left(S_{51}-i S_{52}\right)-\left(p_{1}+i p_{2}\right)\left(S_{53}-i S_{54}\right)\right], \\
\bar{Q}_{2}=\frac{1}{\sqrt{\left(\eta+p_{3}\right)}}\left[\left(p_{1}-i p_{2}\right)\left(S_{51}-i S_{52}\right)+\left(\eta+p_{3}+p_{0}\right)\left(S_{53}-i S_{54}\right)\right],
\end{gathered}
$$

where

$$
\begin{gathered}
p_{0}^{2}=\mathbf{p}^{2}-\eta^{2} \quad \tilde{S}_{12}=\tilde{J}_{12}+\frac{1}{2}\left(S_{12}+S_{43}\right), \\
\tilde{S}_{01}=\tilde{J}_{01}+\frac{1}{2}\left(S_{13}+S_{42}\right), \quad \tilde{S}_{02}=\tilde{J}_{02}+\frac{1}{2}\left(S_{32}+S_{41}\right),
\end{gathered}
$$

$\tilde{J}_{\alpha \beta}$ are basis elements of the algebra $\mathrm{AO}(1,2)(6.4), S_{k l}$ are basis elements of the algebra $\mathrm{AO}(2,3)$ with the metric tensor $(6.5)$, besides $\left[\tilde{J}_{\alpha \beta}, S_{k l}\right]=0$.

\section{COVARIANT REPRESENTATIONS}

Here we present a special realization of representations of the PPSA when the Poincaré group generators have the form

$$
P_{\mu}=p_{\mu}, \quad J_{\mu \nu}=x_{\mu} p_{\nu}-x_{\nu} p_{\mu}+S_{\mu \nu}
$$

with $S_{\nu \sigma}$ being numerical matrices. Such a realization ( when the "spin part" $S_{\nu \sigma}$ of generators commutes with "orbital part" $\left.x_{\nu} p_{\sigma}-x_{\sigma} p_{\nu}\right)$ can have better perspectives in physics then realizations considered in the above.

We choose $S_{\nu \sigma}$ in the form

$$
S_{a b}=\varepsilon_{a b c} S_{c}, \quad S_{0 a}=i S_{a},
$$

where $S_{a}$ are matrices defined in (4.15). Then the corresponding parasupercharges are

$$
\begin{gathered}
Q_{1}=\sqrt{2 M}\left(-S_{51}+i S_{52}\right), \quad Q_{2}=\sqrt{2 M}\left(S_{53}-i S_{54}\right), \\
\bar{Q}_{1}=\sqrt{\frac{2}{M}}\left[\left(p_{3}-p_{0}\right)\left(S_{51}+i S_{52}\right)+\left(p_{1}+i p_{2}\right)\left(S_{53}+i S_{54}\right)\right], \\
\bar{Q}_{2}=\sqrt{\frac{2}{M}}\left[\left(p_{0}+p_{3}\right)\left(S_{53}+i S_{54}\right)+\left(p_{1}-i p_{2}\right)\left(S_{51}+i S_{52}\right)\right] .
\end{gathered}
$$


To obtain the realizations (7.1)-(7.3) it is sufficient to use the transformation (7.4), (7.5) given below. Moreover, it is easy to verify that the operators (7.1)-(7.3) satisfy the relations $(2.1)-(2.3)$, i.e., realize a representations of this algebra. Besides, if we assume $P_{\nu} P^{\nu}=M^{2}>0, p_{0}=\left(p^{2}+M^{2}\right)^{1 / 2}$, and the matrices $S_{\nu \sigma}, j_{a}$ of (4.15), (7.2), (7.3) have the form (4.16), then this representation is irreducible and belongs to class I. Indeed, the corresponding operators (7.1)-(7.3) reduces to the form (4.18), (4.19) using the transformation

$$
\begin{aligned}
& J_{\mu \nu} \rightarrow U J_{\mu \nu} U^{-1}, \quad P_{\mu} \rightarrow U P_{\mu} U^{-1}, \\
& Q_{A} \rightarrow U Q_{A} U^{-1}, \quad \bar{Q}_{A} \rightarrow U \bar{Q}_{A} U^{-1},
\end{aligned}
$$

where

$$
U=\exp \left(-\frac{i S_{0 a} p_{a}}{p} \operatorname{arth} \frac{p}{E}\right)
$$

\section{DISCUSSION}

Considering IRs of the PPSA we restricted ourselves to the case of positive values of the Casimir operator $C_{3}=P_{0} /\left|P_{0}\right|$. The case of negative energies can be analized in complete analogy with the above but corresponds to another LPSAs in comparison with (4.14) and (5.9). Moreover, in this case we have

$\mathrm{LPSA}=\mathrm{AO}(3) \oplus \mathrm{AO}(1,4)$

for IRs of class I and

$\mathrm{LPSA}=\mathrm{AE}(2) \oplus \mathrm{AO}(1,2)$

for IRs of class II. The corresponding parsupercharges can be obtained from (4.18), (5.14) by the changes $S_{5 a} \rightarrow i S_{5 a}, j_{\alpha} \rightarrow i j_{\alpha}, a=1,2,3,4, \alpha=1,2$, where $S_{\mu \nu}$ and $j_{a}$ are now basis elements of the algebras $\mathrm{AO}(1,4)$ and $\mathrm{A} 0(1,2)$ correspondingly. They satisfy the relations (4.12) with $\delta_{a b} \rightarrow-g_{a b}$, where $g_{11}=g_{22}=g_{33}=g_{44}=-g_{55}=-1$ and $g_{11}=g_{22}=-g_{33}=-1$.

Thus, we have described all possible (up to equivalence) IRs of the PPSA. Here we discuss possible physical interpretations of them.

We start with IRs of class I. First, let us discuss the spin contents of these representations. To make this we reduce them to representations of the Poincaré algebra $\operatorname{AP}(1,3)$ (which is a subalgebra of the PPSA).

Let us restrict ourselves to the case $j=0$ (refer to (4.15), (4.7)). Calculating the corresponding Casimir operator $C=W_{\nu} W^{\nu}$ for the subalgebra $\operatorname{AP}(1,3)$ we obtain from $(4.17),(4.15)$

$$
W_{\mu} W^{\mu}=M^{2} \mathbf{S}^{2}, \quad \mathbf{S}=\left(S_{1}, S_{2}, S_{3}\right),
$$


where

$$
S_{a}=\frac{1}{2}\left(\frac{1}{2} \varepsilon_{a b c} S_{b c}+S_{4 a}\right)
$$

and $S_{a b}, S_{4 a}$ belong to the IR $D\left(n_{1}, n_{2}\right)$ of the algebra $\mathrm{AO}(5)$.

The matrices (8.2) realize a reducible representation of the algebra $\mathrm{AO}(3)$. Indeed, reducing the IR $D\left(n_{1}, n_{2}\right)$ to the representations of the algebra $\mathrm{AO}(4)$ $\supset S_{a b}, S_{4 a}(a, b=1,2,3)$, and continuing this reduction to $\mathrm{AO}(3) \supset S_{a}$ of (8.2), we obtain ${ }^{2}$ the following set of eigenvalues for (8.1)

$$
W_{\mu} W^{\mu}=-M^{2} s(s+1), \quad s=\frac{n_{1}+n_{2}}{2}, \frac{n_{1}+n_{2}-1}{2}, \frac{n_{1}+n_{2}-2}{2}, \ldots, 0 .
$$

Moreover, the multiplicity $M_{s}$ of any value of $s$ (i.e., the degeneration of the corresponding eigenvalue $M^{2} s(s+1)$ of $\left.W_{\nu} W^{\nu}\right)$ is given by the following formulae

$$
M_{s}= \begin{cases}\left(n_{1}-n_{2}+1\right)\left(n_{1}+n_{2}+1-2 s\right), & s \geq \frac{n_{1}-n_{2}}{2} \\ \left(2 n_{2}+1\right)(2 s+1), & s<\frac{n_{1}-n_{2}}{2} .\end{cases}
$$

For the case $j \neq 0$ (see (4.7), (4.15)) the possible spin values can be found as a result of summation of the two momenta, i.e., $j$ and $S$ of (8.2). As a result we have instead of (8.3)

$$
s=\frac{n_{1}+n_{2}}{2}+j, \frac{n_{1}+n_{2}}{2}+j-1, \ldots s_{0}, s_{0}= \begin{cases}0, & \frac{n_{1}+n_{2}}{2} \geq j, \\ j-\frac{n_{1}+n_{2}}{2}, & \frac{n_{1}+n_{2}}{2}<j .\end{cases}
$$

The corresponding multiplicities can be calculated using the Clebsh-Gordon theorem and bearing in mind (8.4).

In accordance with the above IRs of the PPSA can be set into correspondence with parasupermultiplets of particles with spin described by formulae (8.4), (8.5).

As supermultiplets [2], parasupermultiplets includes both bosons and fermions.

Let us consider some examples of IRs. For $n_{1}=n_{2}=1 / 2$ we come to IRs of the Poincaré superalgebra. Indeed, in this case the corresponding operators $Q_{A}$ and $\bar{Q}_{A}$ of (4.18) satisfy the anticommutation relations (2.4),

\footnotetext{
[18]

${ }^{2}$ For the details connected with IRs of the algebras $\mathrm{AO}(5) \supset \mathrm{AO}(4) \supset \mathrm{AO}(3)$ see, e.g.,
} 
defining supercharges. Moreover, the related formulae (8.3)- (8.5) reduce to the well-known relations (see, e.g., [2])

$$
s=j+\frac{1}{2}, j, j-\frac{1}{2} ; \quad M_{j}=2, M_{j \pm 1}=1
$$

(the expressions for $M_{s}$ follows from (8.4) and the Clebsh-Gordon theorem), giving the spin contents of supermultiplets.

Thus, we had obtained IRs of PSA as a particular (and the simplest) case of our more general problem.

For $n_{1}=n_{2}=p / 2, p=1,2, \ldots$ formulae $(7.1)-(7.3)$ present the realization of generators of the Poincaré parasupergroup, which is equivalent to found in [15]. The distinguishing feature of our approach is that we use the explicit matrix constructions (more precisely, IRs of the algebra $\mathrm{AO}(5)$ ) instead of the paragrassmanian variables and their derivatives applied in [15]. The last, of course, admit matrix realizations, and vice versa, our results can be reformulated using the concept of parasuperfield [15].

Consider IRs of class II with discrete spins. The corresponding basis elements are present in (5.14), (5.16).

The considered representations are reducible with respect to the subalgebra $\operatorname{AP}(1,3)$. Indeed, calculating the additional Casimir operator of the $\operatorname{AP}(1,3)$

$$
C=\frac{J_{12} p_{3}+J_{31} p_{2}+J_{23} p_{1}}{p}=\lambda-\frac{1}{2} j-\frac{1}{2} j_{3},
$$

we find that its eigenvalues $\bar{\lambda}$ (associated with helicities of particles) are

$$
\bar{\lambda}=\lambda, \lambda-\frac{1}{2}, \lambda-1, \ldots, \lambda-j .
$$

Thus, the corresponding parasupermultiplets includes $2 j+1$ particles, both bosons and fermions, whose helicities are given in (8.7).

For $j=1 / 2$ we again come to IRs of PSA which is a particular case of more general object, i.e., the Poincaré parasuperalgebra.

Using the transformations found in [21] it is possible to find realizations of IRs of the PPSA beeing uniform for any class I-III of (3.6a). Such realizations are unitary equivalent to ones considered in the above.

\section{APPENDIX}


The orthogonal group $\mathrm{O}(5)$ is a set of all linear transformations of the 5dimensional Euclidean space preserving the quadratic form $x_{1}^{2}+x_{2}^{2}+\ldots+x_{5}^{2}$. The Lie algebra of this group is characterized by relations (4.12). Irreducible representation of the algebra $\mathrm{AO}(5)$ are labelled by pairs of numbers $n_{1}$ and $n_{2}$ (simultaneously integer or half integer).

Each representation of the algebra $\mathrm{AO}(5)$ generates a representation of the algebra AO(4). In the Gel'fand-Zetlin basis [18] all the Casimir operators of the subalgebras $\mathrm{AO}(4) \supset \mathrm{AO}(3) \supset \mathrm{AO}(2)$ are diagonal and are characterized by the eigenvalues $m_{1}, m_{2}$, where $n_{1} \geq m_{1} \geq n_{2} \geq m_{2} \geq-n_{2} ; l$, where $m_{1} \geq l \geq\left|m_{2}\right|, m$, where $l \geq m \geq-l$, correspondingly.

Numerating basis elements by multiindex $\xi\left(\begin{array}{lll}m_{1} & & m_{2} \\ & l & \\ & m\end{array}\right)$ we can represent the action of generators in the form $\left(m_{1}\right.$ and $m_{2}$ are fixed)

$$
\begin{aligned}
& S_{21} \xi\left(\begin{array}{lll}
m_{1} & & m_{2} \\
& l & \\
& m &
\end{array}\right)=m \xi\left(\begin{array}{lll}
m_{1} & & m_{2} \\
& l & \\
& m
\end{array}\right) \\
& S_{32} \xi\left(\begin{array}{lll}
m_{1} & & m_{2} \\
& l & \\
& m &
\end{array}\right)=-\frac{i}{2} \sqrt{(l-m)(l+m+1)} \xi\left(\begin{array}{lll}
m_{1} & & m_{2} \\
& l & \\
& m+1
\end{array}\right)+ \\
& +\frac{i}{2} \sqrt{(l-m+1)(l+m)} \xi\left(\begin{array}{lll}
m_{1} & & m_{2} \\
& l & \\
& m-1
\end{array}\right), \\
& S_{43} \xi\left(\begin{array}{lll}
m_{1} & & m_{2} \\
& l & \\
& m
\end{array}\right)= \\
& =\sqrt{\frac{(l+m+1)(l-m+1)\left(m_{1}-l\right)\left(m_{1}+l+2\right)\left(l-m_{2}+1\right)\left(l+m_{2}+1\right)}{(2 l+1)(2 l+3)(l+1)^{2}}}\left(\begin{array}{ccc}
m_{1} & & m_{2} \\
& l+1 & \\
& m
\end{array}\right)+ \\
& +i \frac{m\left(m_{1}+1\right) m_{2}}{(l+1) l} \xi\left(\begin{array}{lll}
m_{1} & & m_{2} \\
& l & \\
& m
\end{array}\right)-
\end{aligned}
$$




$$
\begin{aligned}
& -\sqrt{\frac{(l+m)(l-m)\left(m_{1}-l+1\right)\left(m_{1}+l+1\right)\left(l-m_{2}\right)\left(l+m_{2}\right)}{(2 l+1)(2 l-1) l^{2}}} \xi\left(\begin{array}{lll}
m_{1} & & m_{2} \\
& l-1 \\
& m
\end{array}\right), \\
& S_{54} \xi\left(\begin{array}{lll}
m_{1} & & m_{2} \\
& l & \\
& m &
\end{array}\right)= \\
& =\sqrt{\frac{\left(m_{1}-l+1\right)\left(m_{1}+l+2\right)\left(n_{1}-m_{1}\right)\left(n_{1}+m_{1}+3\right)\left(m_{1}-n_{2}+1\right)\left(m_{1}+n_{2}+2\right)}{\left(m_{1}+m_{2}+1\right)\left(m_{1}+m_{2}+2\right)\left(m_{1}-m_{2}+1\right)\left(m_{1}-m_{2}+2\right)}} \times \\
& \times \xi\left(\begin{array}{ccc}
m_{1}+1 & & m_{2} \\
& l & \\
& m
\end{array}\right)+ \\
& +\sqrt{\frac{\left(l-m_{2}\right)\left(m_{2}+l+1\right)\left(n_{2}-m_{2}\right)\left(n_{2}+m_{2}+1\right)\left(n_{1}-m_{2}+1\right)\left(n_{1}+m_{2}+2\right)}{\left(m_{1}+m_{2}\right)\left(m_{1}+m_{2}+1\right)\left(m_{1}-m_{2}\right)\left(m_{1}-m_{2}+1\right)}} \times \\
& \times \xi\left(\begin{array}{lll}
m_{1} & & m_{2}+1 \\
& l & \\
& m &
\end{array}\right)+ \\
& +\frac{i}{2} \sqrt{\frac{\left(m_{1}+l+1\right)\left(m_{1}-l\right)\left(n_{1}-m_{1}+1\right)\left(n_{1}+m_{1}+2\right)\left(m_{1}-n_{2}\right)\left(m_{1}+n_{2}+1\right)}{\left(m_{1}+m_{2}\right)\left(m_{1}+m_{2}+1\right)\left(m_{1}-m_{2}\right)\left(m_{1}-m_{2}+1\right)}} \times \\
& \times \xi\left(\begin{array}{lll}
m_{1}-1 & & m_{2} \\
& l & \\
& m
\end{array}\right)+ \\
& +\frac{i}{2} \sqrt{\frac{\left(l-m_{2}+1\right)\left(m_{2}+l\right)\left(n_{2}-m_{2}+1\right)\left(n_{2}+m_{2}\right)\left(n_{1}-m_{2}+2\right)\left(m_{2}+n_{1}+1\right)}{\left(m_{1}+m_{2}\right)\left(m_{1}+m_{2}+1\right)\left(m_{1}-m_{2}+2\right)\left(m_{1}-m_{2}+1\right)}} \times \\
& \times \xi\left(\begin{array}{lll}
m_{1} & & m_{2}-1 \\
& l & \\
& m &
\end{array}\right),
\end{aligned}
$$

other generators can be obtain from (4.12).

\section{ACKNOWLEDGMENT.}

We are undebted to Profs. J.Beckers, W.Fushchich and Dr. N.Debergh for stimulating discussions. 


\section{References}

[1] Gol'fand Yu A and Likhtman E P 1971 Lett. JETP 13 452; Ramond P 1971 Phys.Rev. D 3 2415; Neveu A and Schwartz J 1971 Nucl. Phys. B 31 86; Volkov D V and Akulov V P 1972 Lett. JETP 16 621; 1973 Phys.Lett. B 46109.

[2] Fayet P and Ferrara S 1987 Phys. Rept. C 32 250; 1985 Supersymmetry in Physics (North-Holland, Amsterdam), West P 1986 Introduction to Supersymmetry and Supergravity (World Scientific).

[3] Haag R Lopuszanski J T and Sohnius M F 1975 Nucl.Phys. B 8861.

[4] Salam A and Strathdee J 1978 Fortsch. d. Physik 2657.

[5] Witten E 1981 Nucl. Phys. B 188 513; 1982 B 202253.

[6] Piette B Vinet L 1989 Phys. Lett. A 4 2515; D'Hoker E Kostelecky V A and Vinet L 1989 Spectrum generating superalgebras in dynamical groups and spectrum generating algebras ed. Barut A Bohm A and Ne'eman J (Singapore: Word Scientific).

[7] Rubakov V A and Spiridonov V P 1988 Mod. Phys. Lett. A 3.

[8] Wigner E P 1950 Phys. Rev. 77 711; Green H E 1953 Phys. Rev. 90 270; Greenberg O W and Messian A M L 1965 Phys. Rev. B 1381155.

[9] Beckers J and Debergh N 1990 Nucl. Phys. B 340 767; 1991 J. Math. Phys. 32 1808, 1815.

[10] Semenov V V and Chumakov S M 1991 Phys. Lett. B 262 451; Andrianov A A and Ioffe M V 1991 Phys. Lett. B 255 543; Debergh N 1994 J. Phys. A 27 L213; Nikitin A G 1994 Nonlinear Math. Phys. 1202.

[11] Durand S and Vinet L 1990 J. Phys. A 23 3661; Andrianov A A Ioffe M V Spiridonov V P and Vinet L 1991 Phys. Lett. B 272 297, see also references cited therein.

[12] Fushchich W I and Nikitin A G 1987 Symmetries of Maxwell's Equations (Dordrecht: Reidel). 
[13] Beckers J Debergh N and Nikitin A G 1992 J. Math. Phys. 33, 152; 1992 Mod. Phys. Lett. A 71609.

[14] Beckers J Debergh N and Nikitin A G 1993 J. Phys. A 26 L853-L857.

[15] Beckers J Debergh N 1993 J. Mod. Phys. A 8 5041- 5061.

[16] Wess J and Zumino B 1974 Phys. Lett. B 49 52; 1974 Nucl. Phys. B 7039.

[17] Wigner E P 1939 Ann. Math. 40149.

[18] Gel'fand I M Minlos A P and Shapiro Z Ja 1960 Representations of the Rotation and Lorentz Group (Moscow: Fizmatgiz).

[19] Shirokov Ju M 1957 JETP 33 861, 1208.

[20] Barut A Ra̧zka R 1977 Theory of Group Representations and Applications (Warszawa:PWN).

[21] Fushchich W I and Nikitin A G 1994 Symmetries of Equations of Quantum Mechanics (New York: Allerton Press). 